\title{
Associação do risco familiar com saúde bucal, qualidade de vida e variáveis socioeconômicas
}

\author{
Association of familial risk with oral health, quality of life and socioeconomic variables

\section{Asociación del riesgo familiar con salud oral, calidad de vida y variables socioeconómicas}

Fabio Antonio Villa Nova. Secretaria Municipal de Saúde de Tatuí. Tatuí, SP, Brasil. fabio.villanova@yahoo.com.br (Autor correspondente) Gláucia Maria Bovi Ambrosano. Faculdade de Odontologia de Piracicaba (FOP-UNICAMP). Piracicaba, SP, Brasil. glaucia@fop.unicamp.br Stela Márcia Pereira. Universidade Federal de Lavras (UFLA). Lavras, MG, Brasil. stelapereira@dsa.ufla.br Antonio Carlos Pereira. Faculdade de Odontologia de Piracicaba (FOP-UNICAMP). Piracicaba, SP, Brasil. apereira@fopunicamp.br Marcelo de Castro Meneghin. Faculdade de Odontologia de Piracicaba (FOP-UNICAMP). Piracicaba, SP, Brasil. meneghim@fopunicamp.br

\section{Resumo}

Objetivo: avaliar a relação do risco familiar com saúde bucal, qualidade de vida e variáveis socioeconômicas. Métodos: estudo observacional transversal com 311 indivíduos com idade entre 18 e 71 anos, residentes na área de abrangência de quatro unidades de saúde da família (USF), localizadas em dois municípios do estado de São Paulo. Os voluntários foram avaliados de acordo com: (1) situação clínica (CPO-D e necessidade de tratamento), (2) autopercepção de saúde bucal (OHIP-14), (3) qualidade de vida (WHOQOL-BREF), e (4) condição socioeconômica. Para avaliar o risco familiar, utilizou-se a Escala de Coelho. Os dados foram analisados por meio de modelo multinivel formado por componentes fixos (representados pelas variáveis estudadas) e componentes aleatórios (representados pelos bairros e pelas variâncias nos diferentes níveis). Resultados: a média de idade dos participantes foi de 36,7 anos ( $d p=13$ ), com CP0-D médio de 12,9 ( $\mathrm{dp}=7,0)$. A média da Escala de Risco de Coelho entre os voluntários foi de 2,67 com erro padrão de 0,32 . Apresentaram maior escore de risco total os indivíduos de maior idade $(p=0,0486)$, que moravam em casas com mais residentes $(p<0,001)$, com menos anos de escolaridade $(p=0,0137)$, que não possuíam automóvel $(p=0,0048)$ e com maior escore de OHIP-14 ( $p=0,0130)$. Conclusão: a escala de risco familiar associou-se positivamente às variáveis socioeconômicas, e os indivíduos com maior risco familiar apresentaram pior autopercepção da saúde bucal, mas não percebiam uma pior qualidade de vida em geral.

\section{Abstract}

Objective: to assess the relation of familial risk with oral health, quality of life and socioeconomic variables. Methods: cross-sectional observational study encompassing 311 individuals (18 to 71 years old) living in the catchment area of four Family Health Units located in two municipalities of São Paulo state. The participants were evaluated according to: (1) clinical situation (CPO-D and treatment necessity); (2) self-perception oral health (OHIP-14), (3) quality of life (WHOQOL-BREF); and (4) socioeconomic status. The Coelho Scale was used for assessing familial risk. Data were analyzed using a multilevel model formed by fixed components (represented by the studied variables) and random components (represented by the neighborhoods and the variances in the different levels). Results: the participants' average age was 36.7 years old ( $S D=13$ ), and the average CP0-D was 12.9 (SD=7.0). The average of the Coelho's Risk Scale among the volunteers was 2.67 , with a standard error of 0.32 . The participants with higher total risk score were older individuals $(p=0.0486)$ who lived in houses with more residents $(p<0.001)$, who were less educated $(p=0.0137)$, who did not own a vehicle $(\mathrm{p}=0.0048)$, and who had a higher OHIP-14 score $(\mathrm{p}=0.0130)$. Conclusion: the familial risk scale was positively associated with the socioeconomic variables, and the individuals with higher familial risk score had worse self-perception of their oral health. However, they did not perceive themselves as having worse general quality of life.
Palavras-chave:

Saúde Bucal Qualidade de Vida Fatores Socioeconômicos Estratégia Saúde da Família

\section{Keywords:}

Oral Health

Quality of Life

Socioeconomic Factors

Family Health Strategy
Fonte de financiamento: FOP-UNICAMP, Secretaria Municipal de Saúde de Piracicaba-SP e Secretaria Municipal de Saúde de Tatuí-SP.

Parecer CEP: FOP-UNICAMP, 107/2011, 20/10/2011

Conflito de interesses: declaram não haver. Recebido em: 12/07/2014. Aprovado em: 27/10/2014. 


\section{Resumen}

Objetivo: evaluar la relación del riesgo familiar con salud bucal, calidad de vida y las variables socioeconómicas. Métodos: estudio observacional transversal que abarca 311 individuos (18 a 71 años de edad) habitantes en la zona de captación de cuatro Unidades de Salud Familiar ubicadas en dos municipios del estado de São Paulo. Los participantes fueron evaluados de acordo con: (1) situación clínica (necesidad de tratamiento y CPOD); (2) auto percepción de salud bucal (OHIP-14): (3) calidad de vida (WHOQOL-BREF); y (4) nivel socioeconómico. Para medición del riesgo familiar, se utilizó la Escala de Coelho. Los datos fueron analizados mediante el modelo multinivel que consiste de componentes fijos (representados por las variables) y componentes aleatorios (representados por los barrios y las variaciones en los diferentes niveles). Resultados: la edad media de los participantes fue 36,7 años (de = 13), y la media de lo CPO-D fue 12,9 (de =7,0). La media de riesgo de la Escala de Coelho fue 2,67 con un error estándar de $0,32$. Tuvieran puntuaciones de riesgo más altas los individuos de mayor edad $(p=0,0486)$, que vivían en hogares con más residentes ( $p<0,001)$, con menos años de educación ( $p=0,0137)$, que no tenían coche $(p=0,0048)$ y que tuvieran mayor score de 0 HIP-14 ( $p=0,0130)$. Conclusión: la escala de riesgo familiar se asoció positivamente con las variables socioeconómicas y los individuos con mayor riesgo familiar tuvieran una percepción peor de su salud bucal, sin embargo, no se percibirán como que teniendo peor calidad de vida en general.
Palabras clave:

Salud Bucal

Calidad de Vida

Factores Socioeconómicos

Estrategia de Salud Familiar

\section{Introdução}

Ao longo do tempo, novos conceitos de saúde e doença têm sido elaborados, ampliando-se um entendimento mais abrangente sobre os fatores biopsicossociais das doenças. $\mathrm{O}$ indivíduo passou a ser visto, não de forma fragmentada, mas como um todo, uma vez que suas condiçôes de saúde estão relacionadas às suas experiências subjetivas, às suas interpretaçôes de saúde e doença, ao seu bem-estar e ao seu desempenho físico, social e psicológico. Dessa forma, o conceito qualidade de vida tem estado em evidência na área da saúde nos últimos 30 anos. ${ }^{1}$

A saúde é um processo dinâmico que conecta elementos biológicos e sociais, que são afetados pelas experiências anteriores e pelas circunstâncias atuais. A exposição a fatores de risco, em diferentes fases da vida das pessoas, pode diminuir ou aumentar a saúde dos indivíduos. Durante a vida, a saúde é determinada pelas relações familiares, pelos níveis de privação social, por fatores emocionais e comportamentais, bem como por riscos ocupacionais e estresse no trabalho, sendo que os efeitos a longo prazo de cada um desses componentes se combinam para influenciar os resultados da saúde ou da doença. ${ }^{2}$

O Informe Lalonde, documento oficial do Governo Canadense, publicado em 1974 por Marc Lalonde na época em que foi Ministro da Saúde e Bem-Estar do Canadá, é um dos marcos dessa tendência. Esse documento define o conceito do campo da saúde como constituído de quatro componentes: biologia humana, meio ambiente, estilos de vida e organização da atenção à saúde. Tal definição está mais ajustada ao conhecimento construído no âmbito da epidemiologia de doenças não transmissíveis e apresenta uma orientação claramente preventiva. ${ }^{3}$

De acordo com Paulo Nadanovsk, "é admitido que certo nível de doença seja compatível com a qualidade de vida, e a doença é apenas uma das muitas ameaças à saúde". ${ }^{4} \mathrm{Na}$ promoção da saúde, o foco não é a doença, mas sim as estratégias necessárias para se conquistar a saúde e melhorar a qualidade de vida das pessoas e, neste conceito, são levados em conta fatores ambientais e psicológicos, educação, bem-estar, autoestima, felicidade e saúde. ${ }^{1}$

Desse modo, conhecer os determinantes sociais em saúde e suas relações com o processo saúde/doença nos diferentes grupos populacionais é de grande relevância para direcionar o planejamento em saúde pública. Diagnostica-se a situação dos determinantes sociais em saúde por meio de uma lista de indicadores, que devem contemplar as dimensôes econômica, social, ambiental, institucional e de saúde, de forma integrada, o que permite monitorar, de forma sistemática, as mudanças das condiçôes de vida e, consequentemente, da situação de saúde da população. ${ }^{5}$

Ademais, as desigualdades sociais podem levar a fatores de risco comuns a diversas doenças, incluindo as orais. Compreender os determinantes sociais em saúde fornece, assim, as bases teóricas para uma maior integração das atividades de promoção da saúde bucal e da saúde em geral. ${ }^{6}$

$\mathrm{Na}$ odontologia, os indicadores clínicos são muito utilizados para se medir a prevalência das doenças; entretanto, apresentam como limitação o fato de não identificarem a ocorrência de sintomas percebidos pelas pessoas, como dor e desconforto, e de tampouco informarem o impacto da morbidade no bem-estar individual. Além disso, os mesmos não detectam quais indivíduos doentes terão maior probabilidade de procurar atendimento, já que esta demanda é modulada por fatores econômicos, sociais e culturais. Assim, os indicadores clínicos possuem valor limitado para o planejamento e avaliação de programas. Para suprir esta limitação, foram desenvolvidos questionários que mensuram a que grau as doenças influenciam a qualidade de vida dos indivíduos. ${ }^{7}$ 
Os determinantes em saúde bucal, a forma como as pessoas a percebem e as desigualdades em saúde são, portanto, fenômenos complexos, sendo necessárias novas ferramentas para auxiliar na sua compreensão. Por meio dessas ferramentas é possível planejar as açôes de saúde bucal, principalmente as de cunho preventivo, antecipando seus desfechos e mantendo o foco na saúde. Um sistema de classificação de risco familiar que contemple os determinantes sociais em saúde pode ajudar a organizar a demanda em uma Unidade de Saúde da Família (USF), permitindo identificar os grupos mais vulneráveis às doenças. Ante o exposto, o presente estudo tem por objetivo avaliar a relação do risco familiar com saúde bucal, qualidade de vida e variáveis socioeconômicas.

\section{Métodos}

\section{Delineamento do estudo}

Trata-se de um estudo observacional, transversal, com indivíduos que procuraram quatro Unidades de Saúde da Família de dois municípios localizados no interior do estado de São Paulo, para algum atendimento de rotina. Todos os participantes residiam na área de abrangência das USF's onde foi realizado o estudo, sendo três localizadas em Piracicaba nos bairros Sol Nascente, $1^{\circ}$ de Maio e Boa Esperança e uma localizada no município de Tatuí no bairro Jardim Gonzaga, possuindo essas localidades diferentes realidades sociais. ${ }^{8,9}$

O poder do teste foi calculado pelo aplicativo SAS Power and Sample Size versão 3.1 e pelo programa Epi Info versão 7, resultando em um poder acima de 0,80 para o nível de significância de 0,05, ou seja, o tamanho da amostra foi suficiente para se atingirem os objetivos propostos. A amostra compreendeu indivíduos maiores de 18 anos, pertencentes às famílias da área de abrangência das USF's, de ambos os sexos.

Os indivíduos foram abordados nas USF's e convidados a participar da pesquisa. Após a explicação dos objetivos da mesma, apresentou-se um termo de consentimento livre e esclarecido (TCLE) aos voluntários por meio do qual os mesmos concordavam em participar da pesquisa. Foram excluídos da amostra os indivíduos que se recusaram a assinar o TCLE, os menores de 18 anos, aqueles que estavam incapacitados de responder aos questionários e que não residiam na área de abrangência das unidades de saúde.

A coleta dos dados nas quatro USF's, nos dois municípios, teve a duração de 10 meses. Após a autorização, os participantes tiveram os seus dados gerais anotados e foram classificados de acordo com o risco familiar: ${ }^{10} 33$ foram classificados como R1 (risco mínimo), 15 como R2 e 16 como R3 (risco máximo); 247 não pertenciam a nenhum grupo de risco.

\section{A escala de risco de Coelho}

A escala de risco de Coelho foi desenvolvida com o intuito de se estabelecerem prioridades no atendimento domiciliar e na atenção da população adscrita a uma USF. Nesta escala, são aproveitados os dados da Ficha A do SIAB, a qual é preenchida nas primeiras visitas que o agente comunitário de saúde (ACS) faz às famílias de sua comunidade. Deve ser preenchida uma ficha por família e os dados devem ser atualizados sempre que houver alteração. Para elaborar essa escala, elegem-se sentinelas para avaliação das situaçóes a que as famílias possam estar expostas no dia a dia; a partir disso, realiza-se a pontuação de risco familiar (Quadro 1). Com a aplicação da escala em todas as famílias, a equipe passa a ter maior compreensão sobre a relação entre os determinantes de saúde e as situações vividas por elas. Além disso, a escala de Coelho oferece subsídios para a equipe destinar tempo e metodologias de intervençóes diferenciadas, conforme os riscos apresentados pelas famílias de seu território de abrangência, buscando-se criar uma agenda de prioridades de acordo com o princípio da equidade. ${ }^{10}$

\section{Outros instrumentos e medidas}

Os participantes foram avaliados clinicamente (CPO-D e necessidade de tratamento), e entrevistados por meio de três questionários, sendo um sobre a auto percepção em saúde bucal (OHIP-14) e outro sobre qualidade de vida (WHOQOL-BREF), ambos validados no Brasil, ${ }^{11,12}$ e um sobre avaliaçáo socioeconômica, validado por Pereira et al. ${ }^{13}$ 
Quadro 1. Avaliação de risco familiar de Coelho.

\begin{tabular}{|c|c|c|}
\hline \multicolumn{2}{|c|}{ Dados da Ficha A } & Escore \\
\hline \multicolumn{2}{|l|}{ Acamado } & 3 \\
\hline \multicolumn{2}{|l|}{ Deficiência física } & 3 \\
\hline \multicolumn{2}{|l|}{ Deficiência mental } & 3 \\
\hline \multicolumn{2}{|c|}{ Baixas condições de saneamento } & 3 \\
\hline \multicolumn{2}{|c|}{ Desnutrição (Grave) } & 3 \\
\hline \multicolumn{2}{|l|}{ Drogadição } & 2 \\
\hline \multicolumn{2}{|l|}{ Desemprego } & 2 \\
\hline \multicolumn{2}{|l|}{ Analfabetismo } & 1 \\
\hline \multicolumn{2}{|l|}{ Menor de seis meses } & 1 \\
\hline \multicolumn{2}{|l|}{ Maior de 70 anos } & 1 \\
\hline \multicolumn{2}{|c|}{ Hipertensão Arterial Sistêmica } & 1 \\
\hline \multicolumn{2}{|l|}{ Diabetes Mellitus } & 1 \\
\hline \multirow[t]{3}{*}{ Relação Morador/cômodo } & Se maior que 1 & 3 \\
\hline & Se igual a 1 & 2 \\
\hline & Se menor que 1 & 0 \\
\hline
\end{tabular}

Escore 5 ou $6=(\mathrm{R} 1)$; Escore 7 ou $8=(\mathrm{R} 2)$; Maior que $9=(\mathrm{R} 3)$.

Os dados sobre a experiência de cárie e necessidade de tratamento em função da cárie dentária obedeceram aos critérios preconizados pela Organização Mundial de Saúde. ${ }^{14}$ Para a realização dos exames, foram utilizados os espelhos bucais planos número 5 e as sondas CPI, conhecidas como "ball-point" ou "sondas da OMS". Todos os exames foram realizados em cadeira odontológica e com luz artificial. Os dados da pesquisa foram coletados por um único examinador previamente calibrado (Kappa> 0,92); para a calibraçáo, foram usadas as mesmas condiçôes encontradas durante os exames com os voluntários.

\section{Análise estatística e variáveis}

Os dados foram analisados por meio de modelo multinível formado por componentes fixos representados pelas variáveis estudadas e componentes aleatórios representados pelos bairros e pelas variâncias nos diferentes níveis de acordo com Zanini. ${ }^{15}$ Os ajustes do modelo multinível foram realizados pelo procedimento PROC MIXED do programa estatístico SAS, de acordo com a metodologia descrita em Singer, ${ }^{16}$ Tellezet al. ${ }^{17}$ e no Help do programa estatístico SAS. ${ }^{18}$

No nível 1, foram consideradas as variáveis relativas aos indivíduos (idade, gênero, CPO-D, necessidade de tratamento, renda, número de residentes na mesma moradia, escolaridade, tipo de habitação, posse de automóvel, tipo de água que consome na residência, OHIP-14 e WHOQOL-BREF), e no nível 2, as variáveis relativas às cidades (IDH), sendo avaliado o comportamento da variável "escore de risco" como uma função das variáveis preditoras dos níveis 1 e 2 .

Inicialmente, foi ajustado um modelo apenas com o intercepto (modelo 1); a seguir, foram incluídos os efeitos preditores do nível dos indivíduos com OHIP-14 (modelo 2), sendo ajustado também um modelo com os efeitos preditores do nível dos indivíduos com o WHOQOL-BREF (modelo 3). No modelo 4, foram incluídos o OHIP-14 e o WHOQOL-BREF. No modelo 5, a variável do segundo nível (IDH) foi incluída a fim de se avaliar a influência da mesma na explicação da variabilidade do escore total de risco. A qualidade dos ajustes foi avaliada pela convergência do modelo, critérios de AIC, AICC e estatística -2 vezes o logaritmo da função de verossimilhança. O referencial teórico foi utilizado para a seleção das variáveis, e a 'análise de correlação', para avaliar a multicolinearidade.

\section{Aspectos éticos}

Este trabalho foi aprovado pelo Comitê de Ética em Pesquisa (CEP) da FOP-UNICAMP, conforme resolução 196/96, de 10/10/1996 do Conselho Nacional de Saúde do Ministério da Saúde, sob o protocolo no 107/2011. 


\section{Resultados}

A amostra foi composta por 311 indivíduos com idade entre 18 e 71 anos, de ambos os sexos. A média de idade dos entrevistados foi de 36,7 anos (desvio padrão de 13,0 anos), os quais apresentaram, em média, 12,9 dentes cariados, perdidos ou obturados, sendo a maioria mulheres $(67,8 \%)$, com renda familiar mensal de até 2 salários mínimos e até 8 anos de estudo (Tabela 1). Com relaçáo às variáveis contextuais da amostra, $68 \%$ dos conglomerados apresentaram IDH acima de 0,8, mas com índice de analfabetismo superior a 68\% (Tabela 2).

Analisando-se os resíduos (Tabela 3), considerados como efeitos aleatórios, em todos os modelos pode-se observar que a variação entre os voluntários foi maior do que a variação entre os bairros, sendo que a variação entre os bairros, quanto ao risco, não foi significativa em nenhum dos modelos. Pelo coeficiente de correlação intraclasse, pode-se afirmar que a variação entre os bairros representou aproximadamente $9 \%$ da variação total.

Com a inclusão das variáveis do nível individual ao OHIP-14, modelo 2, observa-se que apresentam maior escore de risco tota $1^{10}$ os indivíduos de maior idade $(\mathrm{p}=0,0544)$, que moravam em casas com mais residentes $(\mathrm{p}<0,001)$, com menor escolaridade $(\mathrm{p}=0,0107)$, que não possuíam automóvel $(\mathrm{p}=0,0048)$ e com maior escore de OHIP-14 ( $\mathrm{p}=0,010)$.

Tabela 1. Características da amostra quanto as variáveis do nível 1 (características dos indivíduos).

\begin{tabular}{|c|c|c|c|}
\hline Variável & & Média & Desvio padrão \\
\hline Idade & & 36,7 & 13,0 \\
\hline CPOD & & 12,9 & 7,0 \\
\hline OHIP14 & & 5,57 & 4,90 \\
\hline \multirow[t]{2}{*}{ WHOQOL } & & 14,63 & 2,26 \\
\hline & Categoria & Frequência & $\%$ \\
\hline \multirow[t]{2}{*}{ Sexo } & Feminino & 217 & $67,8 \%$ \\
\hline & Masculino & 94 & $30,2 \%$ \\
\hline \multirow[t]{2}{*}{ Necessidade de tratamento } & Não & 166 & $53,4 \%$ \\
\hline & Sim & 145 & $46,6 \%$ \\
\hline Renda da família & Até 1 & 41 & $13,2 \%$ \\
\hline \multirow[t]{6}{*}{ (em salários mínimos) } & De 1 a 2 & 94 & $30,2 \%$ \\
\hline & De 2 a 3 & 82 & $26,4 \%$ \\
\hline & De 3 a 4 & 47 & $15,1 \%$ \\
\hline & De 4 a 5 & 19 & $6,1 \%$ \\
\hline & De 5 a 6 & 11 & $3,5 \%$ \\
\hline & Maior que 6 & 17 & $5,5 \%$ \\
\hline \multirow[t]{6}{*}{ Residentes na casa } & Até 2 pessoas & 46 & $14,8 \%$ \\
\hline & 3 pessoas & 68 & $21,9 \%$ \\
\hline & 4 pessoas & 85 & $27,3 \%$ \\
\hline & 5 pessoas & 64 & $20,6 \%$ \\
\hline & 6 pessoas & 20 & $6,4 \%$ \\
\hline & $>6$ pessoas & 28 & $9,0 \%$ \\
\hline \multirow[t]{6}{*}{ Anos de escolaridade } & Não alfabetizada & 8 & $2,6 \%$ \\
\hline & $1^{\mathrm{a}} \mathrm{à} 4^{\mathrm{a}}$ & 66 & $21,2 \%$ \\
\hline & $5^{\mathrm{a}}$ à $8^{\mathrm{a}}$ & 117 & $37,6 \%$ \\
\hline & Ensino médio completo & 101 & $32,5 \%$ \\
\hline & Superior incompleto & 10 & $3,2 \%$ \\
\hline & Superior completo & 9 & $2,9 \%$ \\
\hline \multirow[t]{2}{*}{ Habitação } & Própria & 160 & $51,4 \%$ \\
\hline & Não própria & 151 & $48,6 \%$ \\
\hline \multirow[t]{2}{*}{ Posse de automóvel } & Não & 139 & $44,7 \%$ \\
\hline & Sim & 172 & $55,3 \%$ \\
\hline \multirow[t]{2}{*}{ Tipo de água } & Torneira ou filtro & 186 & $59,8 \%$ \\
\hline & Outras & 125 & $40,2 \%$ \\
\hline
\end{tabular}


Tabela 2. Distribuição de frequências das variáveis do nível 2 (características dos conglomerados).

\begin{tabular}{cccc}
\hline Variável & Categoria & Frequência & $\%$ \\
\hline Bairro & Sol Nascente - Piracicaba & 157 & $50,5 \%$ \\
& $1^{0}$ de Maio - Piracicaba & 40 & $12,9 \%$ \\
& Boa Esperança - Piracicaba & 15 & $4,8 \%$ \\
IDH & Jardim Gonzaga - Tatuí & 99 & $31,8 \%$ \\
& 0,836 - Piracicaba & 212 & $68,2 \%$ \\
Índice de analfabetismo & $0,794-$ Tatuí & 99 & $31,8 \%$ \\
& De 3,05 a 6,55\% - Piracicaba & 212 & $68,2 \%$ \\
& $11,44 \%$ - Tatuí & 99 & $31,8 \%$ \\
\hline
\end{tabular}

Tabela 3. Modelo multinível para escore de risco, segundo variáveis do contexto individual, escala de risco familiar e os indicadores 0HIP-14 e WHOQOL-BREF.

\begin{tabular}{|c|c|c|c|c|c|c|}
\hline & \multicolumn{2}{|c|}{ Modelo 1} & \multicolumn{2}{|c|}{ Modelo 2} & \multicolumn{2}{|c|}{ Modelo 3} \\
\hline & \multicolumn{2}{|c|}{ Só o intercepto } & \multicolumn{2}{|c|}{ OHIP-14 } & \multicolumn{2}{|c|}{ WHOQOL-BREF } \\
\hline & Estimativa (SE) & $\mathrm{p}$-valor & Estimativa (SE) & $\mathrm{p}$-valor & Estimativa (SE) & $\mathrm{p}$-valor \\
\hline \multicolumn{7}{|l|}{ Variáveis do nível 1 (indivíduo) } \\
\hline Intercepto & $2,67(0,32)$ & 0,0036 & $2,50(1,32)$ & 0,1553 & $4,14(1,51)$ & 0,0713 \\
\hline Idade & & & $0,03(0,02)$ & 0,0544 & $0,03(0,02)$ & 0,0556 \\
\hline Sexo & & & $0,28(0,30)$ & 0,3587 & $0,17(0,30)$ & 0,5761 \\
\hline CPOD & & & $-0,04(0,03)$ & 0,1745 & $-0,02(0,02)$ & 0,3735 \\
\hline Necessidade de tratamento & & & $-0,11(0,30)$ & 0,7171 & $-0,37(0,28)$ & 0,1940 \\
\hline Renda & & & $-0,10(0,10)$ & 0,3280 & $-0,11(0,10)$ & 0,2579 \\
\hline Residentes & & & $0,86(0,10)$ & $<0,001$ & $0,89(0,10)$ & $<0,001$ \\
\hline Escolaridade & & & $-0,40(0,16)$ & 0,0107 & $-0,40(0,16)$ & 0,0136 \\
\hline Habitação & & & $-0,19(0,28)$ & 0,5097 & $-0,17(0,29)$ & 0,5598 \\
\hline Automóvel & & & $-0,82(0,29)$ & 0,0048 & $-0,90(0,29)$ & 0,0021 \\
\hline Tipo de água & & & $-0,38(0,30)$ & 0,2009 & $-0,50(0,30)$ & 0,0977 \\
\hline OHIP-14 & & & $0,08(0,03)$ & 0,010 & & \\
\hline WHOQOL-BREF & & & & & $-0,04(0,06)$ & 0,4970 \\
\hline \multicolumn{7}{|l|}{ Variáveis do nível 2 (cidades) } \\
\hline \multicolumn{7}{|l|}{$\mathrm{IDH}$} \\
\hline \multicolumn{7}{|l|}{ Variâncias } \\
\hline Variância entre bairros & $0,26(0,36)$ & 0,2377 & $0,13(0,24)$ & 0,2882 & $0,13(0,23)$ & 0,2787 \\
\hline Variância entre voluntários dentro da cidade & $7,52(0,61)$ & $<0,0001$ & $5,41(0,44)$ & $<0,0001$ & $5,52(0,45)$ & $<0,0001$ \\
\hline
\end{tabular}

SE-Erro Padrão

No modelo 3, foi retirada a variável OHIP-14 e incluída a WHOQOL-BREF podendo-se observar que apresentaram maior escore de risco total ${ }^{10}$ os indivíduos com maior idade $(\mathrm{p}=0,0556)$, que moravam em casas com mais residentes $(\mathrm{p}<0,001)$, com menos anos de escolaridade ( $\mathrm{p}=0,0136)$ e que não possuíam automóvel ( $\mathrm{p}=0,0021)$, não sendo observada diferença significativa para a qualidade de vida medida pela ferramenta WHOQOL-BREF.

Verificou-se pelo modelo 4, Tabela 4, com a inclusão do OHIP-14 e o WHOQOL-BREF, que apresentaram maior escore de risco total ${ }^{10}$ os indivíduos de maior idade ( $\left.\mathrm{p}=0,0517\right)$, que moravam em casas com mais residentes ( $\left.<<0,001\right)$, com menos anos de escolaridade ( $\mathrm{p}=0,0010)$, que não possuíam automóvel ( $\mathrm{p}=0,0050)$ e com maior escore de OHIP-14 ( $\mathrm{p}=0,0127)$.

Quando incluída a variável IDH, modelo 5, o resultado foi confirmado, uma vez que apresentaram maior escore de risco tota $~^{10}$ os indivíduos de maior idade $(\mathrm{p}=0,0486)$, que moravam em casas com mais residentes $(\mathrm{p}<0,001)$, com menos anos de escolaridade ( $\mathrm{p}=0,0137)$, que não possuíam automóvel ( $\mathrm{p}=0,0048)$ e com maior escore de OHIP-14 ( $\mathrm{p}=0,0130)$. 
Tabela 4. Modelo multinivel para escore de risco, segundo variáveis do contexto individual e do modelo completo.

\begin{tabular}{|c|c|c|c|c|}
\hline & \multicolumn{2}{|c|}{ Modelo 4} & \multicolumn{2}{|c|}{ Modelo 5} \\
\hline & \multicolumn{2}{|c|}{ (OHIP-14 e WHOQOL-BREF) } & \multicolumn{2}{|c|}{ Modelo completo } \\
\hline & Estimativa (SE) & $\mathrm{p}$-valor & Estimativa (SE) & $\mathrm{p}$-valor \\
\hline \multicolumn{5}{|l|}{ Variáveis do nível 1 (indivíduo) } \\
\hline Intercepto & $2,11(1,70)$ & 0,3044 & $0,21(4,97)$ & 0,7900 \\
\hline Idade & $0,03(0,02)$ & 0,0517 & $0,03(0,02)$ & 0,0486 \\
\hline Sexo & $0,27(0,30)$ & 0,3771 & $0,25(0,31)$ & 0,4140 \\
\hline CPOD & $-0.04(0,03)$ & 0,1682 & $-0,04(0,02)$ & 0,1678 \\
\hline Necessidade de tratamento & $-0,09(0,30)$ & 0,7450 & $-0,08(0,30)$ & 0,3211 \\
\hline Renda & $-0,10(0,10)$ & 0,3119 & $-0,10(0,10)$ & 0,3211 \\
\hline Residentes & $0,86(0,10)$ & $<0,001$ & $0,86(0,10)$ & $<0,001$ \\
\hline Escolaridade & $-0,41(0,16)$ & 0,0010 & $-0,40(0,16)$ & 0,0137 \\
\hline Habitação & $-0,18(0,28)$ & 0,5208 & $-0,16(0,29)$ & 0,5835 \\
\hline Automóvel & $-0.82(0,29)$ & 0,0050 & $-0,82(0,29)$ & 0,0048 \\
\hline Tipo de água & $-0,38(0,30)$ & 0,2091 & $-0,38(0,30)$ & 0,2140 \\
\hline OHIP-14 & $0,09(0,04)$ & 0,0127 & $0,08(0,04)$ & 0,0130 \\
\hline WHOQOL-BREF & $0,03(0,07)$ & 0,7154 & $0,02(0,07)$ & 0,7265 \\
\hline \multicolumn{5}{|l|}{ Variáveis do nível 2 (cidades) } \\
\hline IDH & & & $2,17(18,27)$ & 0,9163 \\
\hline \multicolumn{5}{|l|}{ Variâncias } \\
\hline Variância entre bairros & $0,13(0,24)$ & 0,2892 & $0,35(0,54)$ & 0,2628 \\
\hline Variância entre voluntários dentro de cidade & $5,43(0,45)$ & $<0,0001$ & $5,42(0,45)$ & $<0,0001$ \\
\hline
\end{tabular}

SE-Erro Padrão

\section{Discussão}

A medicina contemporânea tem maximizado seus esforços para prolongar a vida, além de melhorar a qualidade de vida relacionada à saúde. Dessa forma, a avaliação do estado funcional de um paciente utilizando instrumentos de pesquisa padronizados é um item relevante para o tratamento e o cuidado, pressupondo-se que a condição primária para o alcance da qualidade de vida seja a saúde. ${ }^{19}$

Ante o exposto, este estudo buscou estudar a relação da qualidade de vida percebida, sob a ótica de dois instrumentos preconizados pela literatura, e das condiçóes bucais experimentadas com o risco familiar dos indivíduos.

Os resultados destacam que os indivíduos que pertenciam às famílias classificadas como sendo de maior risco ${ }^{10}$ tinham uma pior autopercepçáo de saúde bucal do que a qualidade de vida geral, visto que a qualidade de vida relacionada à saúde bucal avaliada por meio do indicador OHIP-14 associou-se positivamente à escala de risco utilizada para a análise dos dados. Entretanto, o instrumento que avalia a qualidade de vida geral, o WHOQOL-BREF não foi associado a esta variável. Tais achados apontam para o entendimento de que os impactos funcionais e psicológicos dos problemas bucais parecem ser táo importantes, se não mais, quanto os indicadores clínicos, ao se estimarem as necessidades de tratamento odontológico. ${ }^{20}$

A necessidade de tratamento e o CPO-D são indicadores clínicos de cárie dentária e não foram associados ao desfecho estudado de forma isolada; contudo, houve diferença estatística significante para o indicador OHIP-14 e as variáveis individuais. Tais achados estão de acordo com Bortoli ${ }^{21}$ e Daly. ${ }^{22}$

A escolaridade apresentou associação significativa com o risco total. Tal achado corrobora com a literatura quanto à associação entre escolaridade e problemas de saúde, uma vez que a maior escolaridade pode estar associada a um maior nível de renda e a mais informação, o que determina maior busca pelos serviços odontológicos e resolução dos problemas. ${ }^{1,23,24}$

Quanto à avaliação de risco, observou-se que a variação entre os voluntários dentro dos bairros foi maior do que a variação entre os bairros, uma vez que a variação entre os bairros não foi significativa em nenhum dos modelos. Esta variação individual nos modelos para o escore de risco corrobora com a literatura que relata que em um mesmo contexto podem existir indivíduos com diferentes situaçóes de risco, visto que o risco individual, a suscetibilidade e a vulnerabilidade às doenças devem ser entendidos como parte de um complexo conjunto de influências que incluem o indivíduo, a família, e a comunidade. ${ }^{5,25,26}$ 
Ademais, a identificação das áreas de privação social, levando em conta os determinantes sociais da saúde e da população, é importante para a saúde pública. Os determinantes sociais que têm sido investigados incluem fatores como a qualidade da moradia, a disponibilidade de transportes públicos, os índices de criminalidade, a iluminação pública, o acesso a parques e a espaços abertos, e o grau de coesão social e de apoio emocional presentes nas relaçóes pessoais. As desvantagens sociais e ambientais podem influenciar diretamente as condiçóes de saúde. ${ }^{27}$ Deste modo, o estudo de instrumentos que possibilitem priorizar as visitas domiciliares, identificando, assim, as famílias em maior situaçáo de vulnerabilidade dentro do processo de trabalho das equipes multiprofissionais inseridas nas USF's é relevante.

Ainda com relação ao risco, no modelo multinível, observou-se que apresentaram maior risco total os indivíduos de maior idade, que moravam em casas com mais residentes e com menos anos de escolaridade. Essas variáveis têm relação com as sentinelas de risco (acima de 70 anos, relação morador-cômodo e analfabetismo, respectivamente) da escala de risco familiar utilizada. ${ }^{10}$ Os dados analisados confirmaram que os indivíduos de maior idade, que viviam em casas com maior número de pessoas, que possuíam menos anos de estudo e que não tinham automóvel, apresentaram maiores escores de risco, o que foi confirmado em todos os modelos.

A busca ativa dos indivíduos propensos a adoecer faz parte das diretrizes da Estratégia Saúde da Família (ESF). Portanto, a equipe da unidade deve ter conhecimento da localizaçáo das famílias vulneráveis do território para o estabelecimento de medidas de promoção da saúde que levem à qualidade de vida desta população. No presente estudo, as variáveis socioeconômicas, que podem influenciar a saúde dos indivíduos, e o instrumento de autopercepção de saúde bucal foram associados positivamente à escala de Risco de Coelho, demonstrando ser este um instrumento válido para a classificação de risco das famílias podendo ser utilizado em unidades de saúde. Na Escala de Coelho ${ }^{10}$, a sentinela de risco "aglomeração familiar" tem grande peso no escore final, pois nesta situação os indivíduos estão mais propensos às doenças infectocontagiosas, bem como a baixas condiçóes de higiene. Estudos sugerem ${ }^{25,28}$ que o critério de avaliação "baixas condiçóes de higiene", que a escala de Risco de Coelho ${ }^{10}$ não contempla, seja adicionado ao item "baixas condiçóes de saneamento".

$\mathrm{Na}$ escala de risco ${ }^{10}$ utilizada neste estudo, foram usados os dados contidos na ficha A do SIAB que devem ser atualizados periodicamente pelos ACS. Há necessidade de maiores esforços para melhorar a capacitação do ACS, visando adequar seu nível de apreensão e conhecimento dos princípios do SUS e da ESF, para que ele possa atuar segundo as diretrizes desse sistema e contribuir efetivamente para sua consolidação. ${ }^{29}$

\section{Conclusão}

No presente estudo, a escala de risco familiar associou-se positivamente às variáveis socioeconômicas, assim como os indivíduos com maior risco familiar apresentaram uma pior autopercepção da saúde bucal, embora não percebessem uma pior qualidade de vida em geral. Portanto, os instrumentos de classificação de risco familiar devem ser adequados às diferentes realidades sociais existentes no território nacional e, para que isso seja efetivo, é necessário que toda equipe multidisciplinar das unidades de saúde receba capacitação técnica adequada para utilizá-los como ferramenta na busca ativa das famílias de risco adstritas às unidades de saúde, assim como no planejamento das atividades da equipe, como forma de garantir maiores recursos e atenção aos indivíduos em situação de vulnerabilidade.

\section{Referências}

1. Mesquita F, Vieira S. Impacto da condição de autoavaliação de saúde bucal na qualidade de vida. RGO. 2009;57(4):401-406.

2. Nicolau B, Marcenes W. How will a life course framework be used to tackle wider socialdeterminants of health? Community Dent OralEpidemiol. 2012 Oct;40(Suppl 2):33-8. http://dx.doi.org/10.1111/j.1600-0528.2012.00717.x

3. Lalonde M. A new perspective on the health of Canadians. Ottawa: Health and Welfare Canada; 1974.

4. Nadanovsk P. Promoção da saúde e a prevenção das doenças bucais. In: Pinto VG. Saúde bucal coletiva. 4a ed. São Paulo: Santos; 2000. p. 293.

5. Sobral A, Freitas CM. Modelo de Organização de Indicadores para operacionalização dos determinantes sócio ambientais da saúde. Saúde Soc. 2010;19(1):35-47. http://dx.doi.org/10.1590/S0104-12902010000100004

6. Watt RG. Social determinants of oral health inequalities: implications for action. Community Dent Oral Epidemiol. 2012 Oct;40(Suppl 2):44-8. http://dx.doi.org/10.1111/j.1600-0528.2012.00719.x 
7. Luiz RR. Epidemiologia e Bioestatística em Odontologia. Ed revista e ampliada. São Paulo: Ateneu; 2008.

8. Instituto de Pesquisa e Planejamento de Piracicaba (IPPLAP). c2015 [acesso em 2012 Sep 06]. Disponível em: www.ipplap.com.br

9. Tatuí. Taxa de analfabetismo por bairro no município de Tatuí- SP. [acesso em 2012 Sep 06]. Disponível em: http://tatui.sp.gov.br/sobre-tatui/dados-gerais

10. Coelho FLG, Savassi LCM. Aplicação da Escala de Risco Familiar como instrumento de priorização das visitas domiciliares. Rev Bras Med Fam Comunidade. 2003 [acesso em 2011 May 01];1(2):19-26. Disponível em: http://www.rbmfc.org.br/rbmfc/article/view/104

11. Oliveira BH, Nadanovsky P. Psychometric properties of the Brazilian version of the Oral Health Impact Profile-short form. Community Dent Oral Epidemiol. 2005;33(4):307-14. http://dx.doi.org/10.1111/j.1600-0528.2005.00225.x

12. Fleck MPA, et al. Aplicação da versão em português do instrumento abreviado de avaliação da qualidade de vida "WHOQOL-bref". Rev Saúde Pública. 2000;34(2):178-183. http://dx.doi.org/10.1590/S0034-89102000000200012

13. Pereira SM, Tagliaferro EP, Ambrosano GM, Cortelazzi KL, MeneghimMC, Pereira AC. Dental caries in 12-year-old schoolchildren and its relationship with socioeconomic and behavioural variables. Oral Health Prev Dent. 2007;5(4):299-306.

14. World Health Organization. Oral health serveys: basic methods. $4^{\text {th }}$ ed. Geneva; 1997.

15. Zanini RR. Modelos multiníveis aplicados ao estudo da mortalidade infantil e no Rio Grande da Sul, Brasil, de 1994 a 2004 [tese]. Rio Grande do Sul: Universidade Federal do Rio Grande do Sul; 2007. Portuguese.

16. Singer JD. Using SAS proc mixed to fit multilevel models, hierarchical models and individual growth models. J Educational Behavioral Statistics. 1998;24(4):323-355. http://dx.doi.org/10.2307/1165280

17. Tellez M, Sohn W, Burt BA, Ismail Al. Assessment of the relationship between neighborhood characteristics and dental caries severity among low-income African-Americans: A Multilevel Approach. J Public Health Dentistry. 2006;66(1):30-36.

http://dx.doi.org/10.1111/j.1752-7325.2006.tb02548.x

18. SAS 9.2 User's Guide, Second Edition. Cary, NC, USA: SAS Institute Inc.; 2010. Disponível em: http://support.sas.com/documentation/cdl/en/statug/63033/HTML/default/viewer.htm\#titlepage.htm

19. Muszalik M, Kedziora-Kornatowska K, Kornatowski T. Functional assessment and health-related quality of life (HRQOL) of elderly patients on the basis of the functional assessment of chronic illness therapy (FACIT)-F questionnaire. Arch Gerontol Geriatr. 2009 Nov-Dec;49(3):404-8. Epub 2009 Jan 28.http://dx.doi.org/10.1016/j.archger.2008.12.007

20. Seremidi K, Koletsi-Kounari H, Kandilorou H. Self-reported and clinically-diagnosed dental needs: determining the factors that affect subjective assessment. Oral Health Prev Dent. 2009;7(2):183-90.

21. Bortoli D, Locatelli FA, Fadel CB, Baldani MH. Associação entre percepção de saúde bucal e indicadores cínicos e subjetivos: estudo em adultos de um grupo de educação continuada da terceira idade. Publ UEPG Ci Biol Saúde, Ponta Grossa. 2003;9(3/4):55-65.

22. Daly B, Newton T, Batchelor P, Jones K. Oral health care needs and oral health-related quality of life (OHIP-14) in homeless people. Community Dent Oral Epidemiol. 2010;38:136-144.http://dx.doi.org/10.1111/j.1600-0528.2009.00516.x

23. Cortellazzi $\mathrm{KL}$ et al. Influência de variáveis socioeconômicas, clínicas e demográfica na experiência de cárie dentária em pré-escolares de Piracicaba, SP. Rev Bras Epidemiol. 2009;12(3):490-500. http://dx.doi.org/10.1590/S1415-790X2009000300017

24. Szpilmanet ARM, Martins FG, Jantorno C, Coutinho Junior EZ, Silva LR, Sylvestre NC, et al. Condição de saúde bucal de crianças de zero a dois anos de idade inseridas na estratégia saúde da família (ESF). Rev Bras Pesquisa Saúde. 2012;14(1):42-48.

25. Nascimento FG, Prado TN, Galavote HS, Maciel PA, Lima R CD, Maciel ELN. Aplicabilidade de uma escala de risco para organização do processo de trabalho com famílias atendidas na Unidade Saúde da Família em Vitória (ES). Ciênc Saúde Coletiva. 2010;15(5):2465-72. http://dx.doi.org/10.1590/S1413-81232010000500021

26. Garcia L, Tabak LA. Global oral health inequalities: the view from a research funder. Adv Dent Res. 2011;23(2). http://dx.doi.org/10.1177/0022034511402015

27. Burt BA. Concepts of risk in dental public health. Community Dent Oral Epidemiol. 2005;33:240-7. http://dx.doi.org/10.1111/j.1600-0528.2005.00231.x

28. Costa CM. A aplicação da escala de risco familiar no Programa de Saúde da Família como estratégia de priorização das visitas domiciliares pelos agentes comunitários de saúde [dissertação]. Rio de Janeiro: Universidade Estácio de Sá; 2009.

29. Gomes KO, Cotta RMM, Cherchiglia ML, Mitre SM, Batista RS. A práxis do agente comunitário de saúde no contexto do Programa Saúde da Família: reflexões estratégicas. Saúde Soc. 2009;18(4):744-755. http://dx.doi.org/10.1590/S0104-12902009000400017 